PROCEEDINGS OF THE AMERICAN MATHEMATICAL SOCIETY

Volume 135, Number 4, April 2007, Pages 1187-1196

S 0002-9939(06)08631-X

Article electronically published on October 27, 2006

\title{
STOCHASTIC PARAMETERIZATION FOR LARGE EDDY SIMULATION OF GEOPHYSICAL FLOWS
}

\author{
JINQIAO DUAN AND BALASUBRAMANYA T. NADIGA
}

(Communicated by Edward C. Waymire)

\begin{abstract}
Recently, stochastic, as opposed to deterministic, parameterizations are being investigated to model the effects of unresolved subgrid scales (SGS) in large eddy simulations (LES) of geophysical flows. We analyse such a stochastic approach in the barotropic vorticity equation to show that (i) if the stochastic parameterization approximates the actual SGS stresses, then the solution of the stochastic LES approximates the "true" solution at appropriate scale sizes; and that (ii) when the filter scale size approaches zero, the solution of the stochastic LES approaches the true solution.
\end{abstract}

\section{Motivation}

The immense number of degrees of freedom in large scale turbulent flows as encountered in the world's oceans and atmosphere makes it impossible to simulate these flows in all their detail in the foreseeable future. On the other hand, it is essential to represent these flows reasonably accurately in Ocean and Atmospheric General Circulation Models (OGCMs and AGCMs) so as to improve the confidence in these model components of the earth's system in an ongoing effort to study climate and its variability (e.g., see [2]). Furthermore, it is very often the case that in highly resolved computations, a rather disproportionately large fraction of the computational effort is expended on the small scales (e.g., see [5]) whereas a large fraction of the energy resides in the large scales (e.g., see [6]). It is for these reasons that the ideas of Large Eddy Simulation (LES) - wherein the large scale unsteady motions driven by specifics of the flow are explicitly computed, but the small (and presumably more universal [7]) scales are modelled - are natural in this context.

Given our interest in large scale geophysical flows with their small vertical to horizontal aspect ratio, we restrict ourselves to two-dimensional or quasi-twodimensional flows. Previous models of the small scales in how they affect the large scales in the momentum equations or equivalently the vorticity equation in incompressible settings have mostly been confined to an enhanced eddy viscosity or nonlinear eddy viscosity like that of Smagorinsky or biharmonic viscosity (e.g., see [8, 9, 10, 11, 12, 13]). Given the nonunique nature of the small scales with respect

Received by the editors October 30, 2005 and, in revised form, November 23, 2005.

2000 Mathematics Subject Classification. Primary 65C20, 60H15, 86A10.

The first author was partly supported by NSF Grants DMS-0209326 \& DMS-0542450.

The second author was partly supported by the LDRD program at Los Alamos National Laboratory. 
to the large scales [5], the aforementioned use of deterministic and dissipative closures seems rather highly restrictive. On the other hand, it would seem desirable to actually represent a population of eddies that satisfy overall constraints of the flow rather than make flow specific parametric assumptions. This has led to recent investigations of the possibility of using stochastic processes to model the effects of unresolved scales in geophysical flows (e.g., see [14, 15]). More recently, subgrid scale (SGS) stresses have been analysed in simple but resolved flows as a possible way to suggest stochastic parameterizations as, e.g., in [3, 4, 30, 31. These efforts have been preceded, of course, by various attempts to model anomalies in geophysical flow systems as linear Langevin equations (e.g., [28, 1, 16, 26]) and the analysis of stochastic models in isotropic and homogeneous three-dimensional turbulence (e.g., [17, 18]).

In this paper, we analyze the stochastic approach to parameterization in the barotropic vorticity equation and show that (i) if the stochastic parameterization approximates the SGS stresses, then the stochastic large eddy solution approximates the "true" solution at appropriate scale sizes; and that (ii) when the filter scale size approaches zero, then the solution of the stochastic LES approaches the true solution. In the next section we present a set of computations that demonstrates the use of stochastic parameterizations and in $\S 3$, we prove the main results on approximation and convergence of LES solutions using stochastic parameterizations.

\section{Stochastic PARAMETERIZATION}

We consider the simple setting of the beta-plane barotropic vorticity equation (equivalently the two-dimensional (2D) quasi-geostrophic (QG) model) [27, 34]:

$$
q_{t}+J(\psi, q)+\beta \psi_{x}=f(x, y, t)+\nu \Delta q-r q,
$$

on a bounded domain $D$ with piecewise smooth boundary $\partial D$. Here the vorticity $q(x, y, t)$ is given in terms of the stream function $\psi(x, y, t)$ by $q=\Delta \psi$. Moreover, $\beta$ is the meridional gradient of the Coriolis parameter, $\nu>0$ the viscous dissipation constant, $r>0$ the Ekman dissipation constant and $f(x, y, t)$ the wind forcing. The forcing $f$ is always assumed to be mean-square integrable both in time and in space. In addition, $\Delta=\partial_{x x}+\partial_{y y}$ is the Laplacian operator in the plane and $J(h, g)=h_{x} g_{y}-h_{y} g_{x}$ is the Jacobian operator. The boundary condition (BC) is $q=0, \psi=0$ on $\partial D$ and the initial condition (IC) is $q(x, y, 0)=q_{0}(x, y)$.

Fine mesh simulations $(q)$ are used to obtain the benchmark solution $\bar{q}$ through convolution with a spatial filter $G_{\delta}(x, y)$, with spatial scale $\delta>0$ :

$$
\bar{q}(x, y, t):=q * G_{\delta} .
$$

We use a Gaussian filter [19], $G_{\delta}(x, y)=\frac{1}{\pi \delta^{2}} e^{-\frac{\left(x^{2}+y^{2}\right)}{\delta^{2}}}$, where $\delta>0$ is the filter size and the filter is such that (1) $q * G_{\delta}$ is infinitely differentiable in space and (2) $q * G_{\delta} \rightarrow q$ as $\delta \rightarrow 0$ in $L^{2}(D)$. Note that the Fourier transform of $G_{\delta}$ is $\widehat{G}_{\delta}\left(k_{1}, k_{2}\right)=e^{-\frac{\delta^{2}\left(k_{1}^{2}+k_{2}^{2}\right)}{4}}$, and that $\widehat{q * G_{\delta}}\left(k_{1}, k_{2}, t\right)=\widehat{G}_{\delta}\left(k_{1}, k_{2}\right) \widehat{q}\left(k_{1}, k_{2}, t\right)$.

On convoluting (2.1) with $G_{\delta}$ the large eddy solution $\bar{q}$ is seen to satisfy

$$
\bar{q}_{t}+J(\bar{\psi}, \bar{q})+\beta \bar{\psi}_{x}=\bar{f}(x, y, t)+\nu \Delta \bar{q}-r \bar{q}+R(q),
$$

where the SGS stress term $R(q)$ is defined as

$$
R(q):=J(\bar{\psi}, \bar{q})-\overline{J(\psi, q)}
$$


Note that since $\overline{\bar{q}} \neq \bar{q}$, the SGS stress term $R(q)$ above is more than the Reynolds stress and the SGS stress is usually further divided into three components, the explicit Leonard stress, and the cross stress and the SGS Reynolds stress that require further modeling. However, for our purposes, we will consider $R(q)$ in its entirety. Since $R(q)$ depends on $q$ as well as $\bar{q}$, the equation (2.3) is not a closed system. We need to model or prescribe $R(q)$ in terms of the resolved quantity $\bar{q}$. On the other hand, $R(q)$ may be explicitly diagnosed from a fully-resolved run, given the filter.

An analysis of $R(q)$ reveals that its time-mean is much smaller than its standard deviation, and that its temporal behavior is highly irregular, leading to the possibility of approximating $R(q)$ by a suitable stochastic process $\sigma(\bar{q}, \omega)$ (defined on a probability space $(\Omega, \mathcal{F}, \mathbb{P})$, with $\omega \in \Omega$, the sample space, $\sigma$-field $\mathcal{F}$ and probability measure $\mathbb{P}$ ). With such a putative stochastic closure, the LES model becomes a random partial differential equation (PDE) for $Q \sim \bar{q}$ :

$$
Q_{t}+J(\Psi, Q)+\beta \Psi_{x}=\bar{f}(x, y, t)+\nu \Delta Q-r Q+\sigma(Q, \omega),
$$

where $Q=\Delta \Psi$ and $\bar{f}(x, y, t):=f * G_{\delta}$, with BC: $Q=0, \Psi=0$ on $\partial D$ and IC: $Q(x, y, 0)=\bar{q}_{0}(x, y)$. Note that when the stochastic process $\sigma(Q, \omega)$ does not depend explicitly on $Q$ (as is the case when for example, either $R(q)$ or some of its statistical properties are used to construct $\sigma(\omega)$ ) we end up with an additive stochastic closure. The more general case of the stochastic closure wherein there is an explicit dependence on the state of the system $Q$, corresponds to a multiplicative stochastic closure.

While one can get an idea of the stochastic forcing to be used to represent the effects of unresolved subgrid scales in the LES runs by analysing resolved runs at the scale of the LES computation, the selection of a specific functional form for the stochastic parameterization is beyond the scope of the present article. We aim at providing a quantitative guide to selecting the stochastic parameterization.

2.1. Numerical experiments. We now briefly present a set of computations using the beta-plane barotropic vorticity equation (2.1) in a rectangular midlatitude basin. Finite differencing in space is used along with Runge-Kutta time stepping, and other details of the setup may be found elsewhere [32. The steady forcing is uniform in the zonal $(x)$ directions and sinusoidal in the meridional $(y)$ direction corresponding to a double-gyre wind forcing. At the parameter values that we are presently considering, the circulation is highly variable, but statistically stationary. We therefore consider long time averages over the attractor in place of ensemble averaging (over $\omega$ ).

Figure 1 shows the contour plots of the time average of the stream function and potential vorticity as they emerge in the resolved computations. A discussion of the phenomenology of this circulation may be found in 32. This simulation is then analysed using a Gaussian filter with a width that is four times the grid spacing of the resolved computations to obtain the SGS stress $R(q)$.

Next we consider a pair of coarse-scale simulations in which the grid spacing is four times that of the resolved computation in both directions. Figure 2 shows the time average of the stream function and potential vorticity as emerges from the coarse-scale computation when $\sigma(Q, \omega)$ in (2.5) is set to zero. The main differences, as compared to the resolved runs, clearly are the absence of the outer gyres in the 

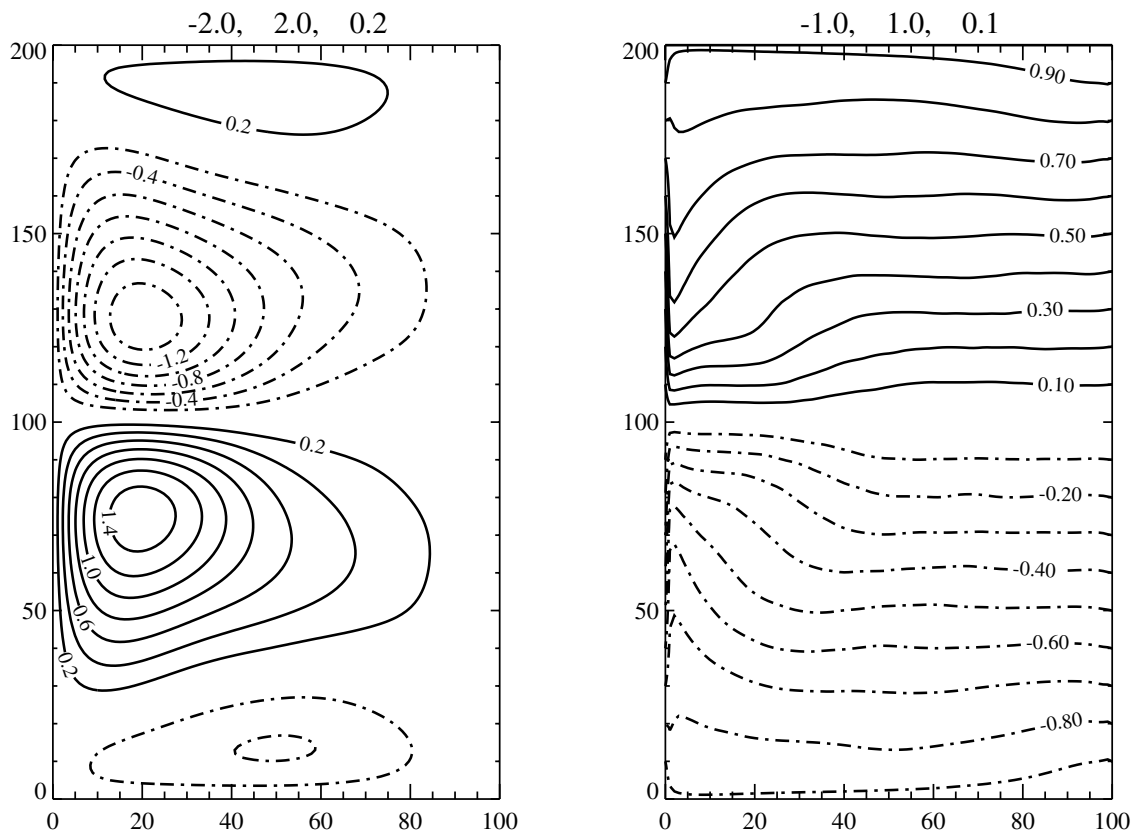

Figure 1. Resolved simulation. Contour plots of the average over the attractor (time-average) of the stream function and potential vorticity. Contour intervals are the same in all the figures and are indicated on top of the figures in the form (min, max, increment).

stream-function field and the large amplitude grid scale oscillation in the potentialvorticity field.

In the next case, we set the statistics of $\sigma(Q, \omega)$ (viz., its spatial and temporal correlation functions, amplitude and probability distribution function) identical to those of $R(q)$ previously diagnosed from the resolved simulation. This we do by using the actual time history of the SGS stress $R(q)$ in a coarse-resolution run in which the initial condition is slightly perturbed from the initial condition of the resolved run from which $R(q)$ is diagnosed. Given the highly chaotic nature of the flow, the effect of the initial perturbation is to quickly lead to a complete decorrelation of the SGS stress-forcing term $R(q)$ from the state of the system $Q$. Thus, the actual time history of the diagnosed SGS stress $R(q)$ supplied to the coarse-resolution run acts effectively as an additive stochastic closure $\sigma(\omega)$ of the SGS stresses in this LES. The time averages of the circulation for this case are shown in Figure 3. Comparing Figure 2 and Figure 1 and Figure 3 and Figure 1, it is clear that the differences between the resolved case and the case with $\sigma(Q, \omega)$ similar to $R(q)$ are much smaller than the differences between the resolved case and the case with $\sigma(Q, \omega)=0$.

These numerical experiments suggest that stochastic parameterizations can provide good representations of subgrid scales. So, the question then is as to how such an approach can be justified.

In an attempt to answer this question, we will prove, following the approaches in 19, 29] in our stochastic context, that, under appropriate conditions on the 

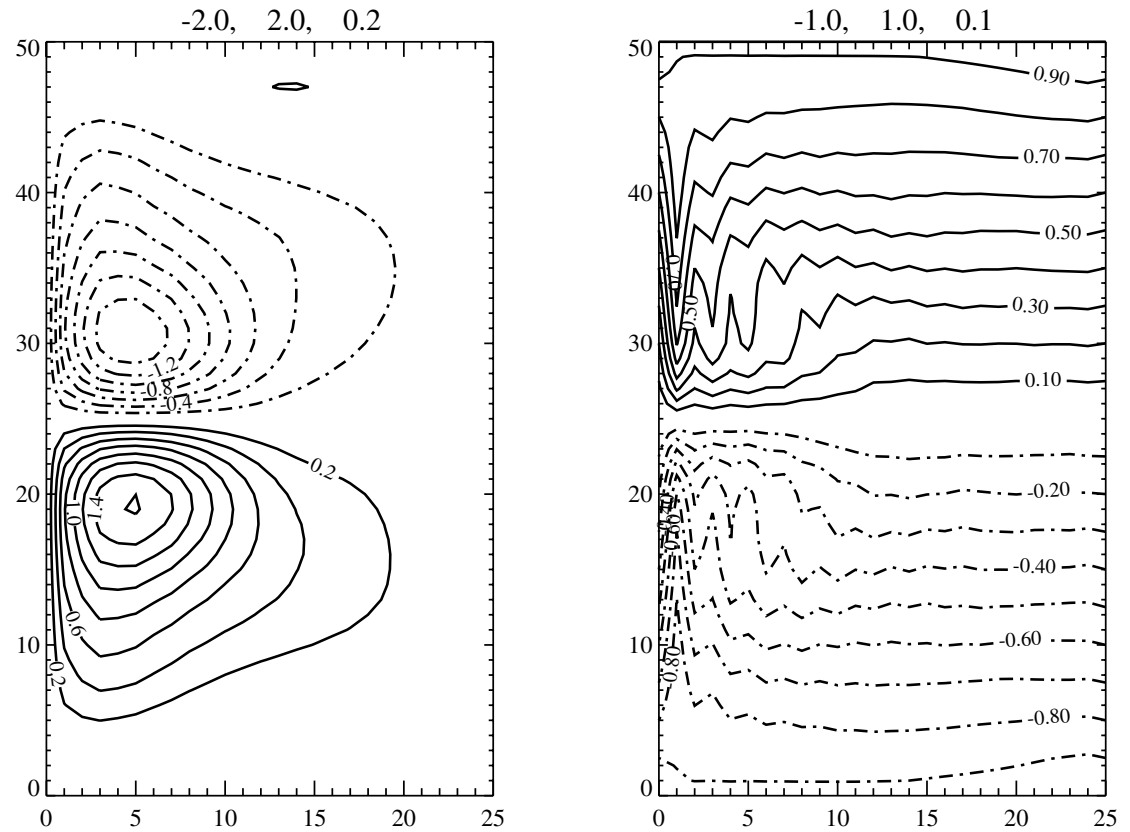

Figure 2. Coarse unresolved simulation. Contour plots of stream function and potential vorticity, as in Figure 1. The stochastic parameterization term $\sigma(Q, \omega)$ is set to 0 .
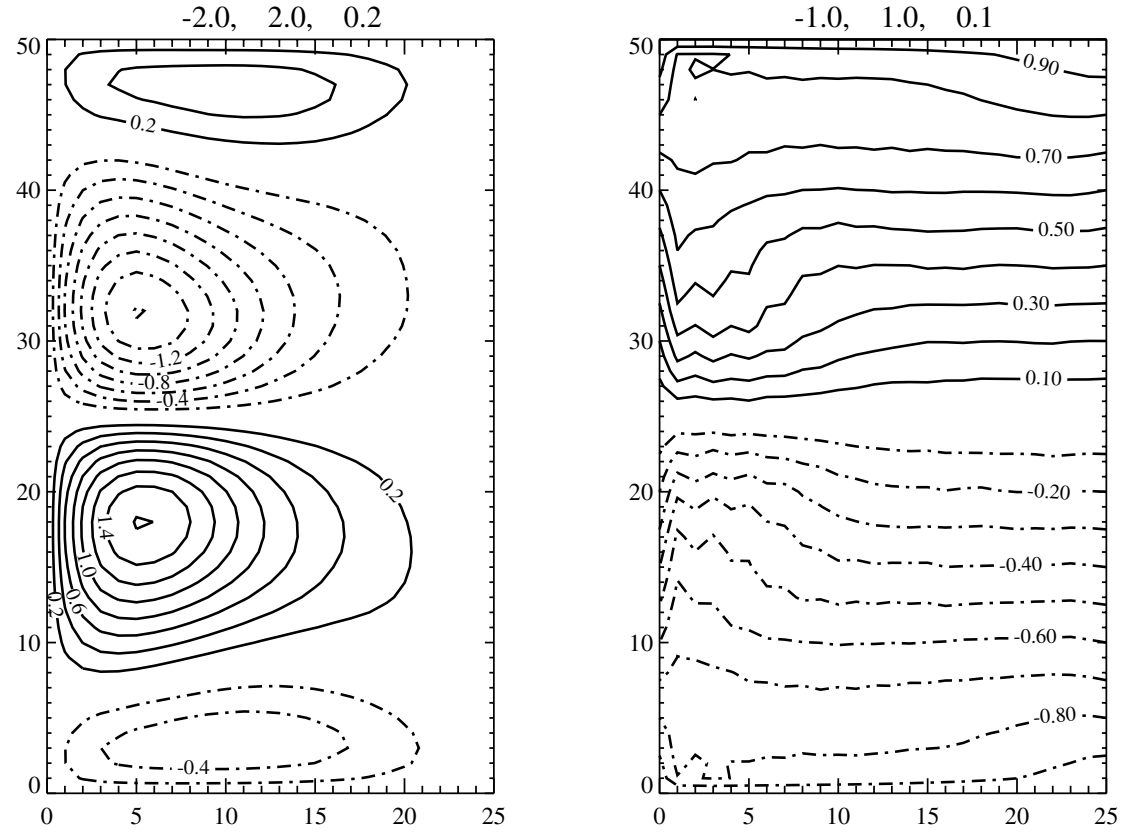

FiguRE 3. LES with stochastic parameterization. Contour plots of the stream function and potential vorticity, as in Figure 1. The statistics of $\sigma(Q, \omega)$ are identical to those of $R(q)$. 
stochastic parameterization (that appear easier to check in our case, cf. Theorem 3.2 part (i) below and Assumption A2 in [29]):

$$
\mathbb{E}\|\bar{q}-Q\|^{2} \leq C\left(\nu, r, q_{0}, T\right) \cdot \mathbb{E} \int_{0}^{T}\|R(q)-\sigma(Q, \omega)\|^{2} d t, \quad 0 \leq t \leq T
$$

where $C(\cdot)>0$ is a constant, $[0, T]$ is the computational time interval, $\mathbb{E}(Z(\omega)):=$ $\int_{\Omega} Z(\omega) d \mathbb{P}(\omega)$, and $\|\cdot\|$ is the (spatial) norm in the space $L^{2}(D)$ of spatially meansquare integrable functions: $L^{2}(D):=\left\{f:\|f\|=\sqrt{\int_{D} f(x, y) d x d y}<\infty\right\}$.

\section{MAIN RESUlTS}

Standard abbreviations $L^{2}=L^{2}(D), H_{0}^{k}=H_{0}^{k}(D), k=1,2, \ldots$, are used for the common Sobolev spaces in fluid mechanics 36, 22, with $\langle\cdot, \cdot\rangle$ and $\|\cdot\|$ denoting the usual (spatial) scalar product and norm, respectively, in $L^{2}(D)$ :

$$
\langle f, g\rangle:=\int_{D} f g d x d y, \quad\|f\|:=\sqrt{\langle f, f\rangle}=\sqrt{\int_{D} f(x, y) d x d y} .
$$

We need the following properties and estimates (see also [25, 20]) of the Jacobian operator $J: H_{0}^{1} \times H_{0}^{1} \rightarrow L^{1}$ :

$$
\begin{array}{r}
\int_{D} J(f, g) h d x d y=-\int_{D} J(f, h) g d x d y, \int_{D} J(f, g) g d x d y=0, \quad \text { and } \\
\left|\int_{D} J(f, g) d x d y\right| \leq\|\nabla f\|\|\nabla g\| \quad \text { for all } f, g, h \in H_{0}^{1}, \\
\left|\int_{D} J(\Delta f, g) \Delta h d x d y\right| \leq \sqrt{\frac{2|D|}{\pi}}\|\Delta f\|\|\Delta g\|\|\Delta h\| \quad \text { for all } f, g, h \in H_{0}^{2} .
\end{array}
$$

In addition, we recall the Poincaré, Young, and Gronwall inequalities below:

$$
\begin{aligned}
& \|g\|^{2}=\int_{D} g^{2}(x, y) d x d y \leq \frac{|D|}{\pi} \int_{D}|\nabla g|^{2} d x d y=\frac{|D|}{\pi}\|\nabla g\|^{2} \quad \text { for } g \in H_{0}^{1}, \\
& A B \leq \frac{\epsilon}{2} A^{2}+\frac{1}{2 \epsilon} B^{2} \text { for } A, B \geq 0 \text { and } \epsilon>0 .
\end{aligned}
$$

Assuming that $y(t) \geq 0, g(t)$ and $h(t)$ are integrable, if $\frac{d y}{d t} \leq g y+h$ for $t \geq t_{0}$, then

$$
y(t) \leq y\left(t_{0}\right) e^{\int_{t_{0}}^{t} g(\tau) d \tau}+\int_{t_{0}}^{t} h(s) e^{-\int_{t}^{s} g(\tau) d \tau} d s, \quad t \geq t_{0} .
$$

Lemma 3.1 ([20]). The quasi-geostrophic motion described by (2.1) satisfies the enstrophy estimate

$$
\begin{aligned}
\|q\|^{2} & \leq\left\|q_{0}\right\|^{2} e^{-2 \alpha t}+\frac{1}{r} \int_{0}^{t}\|f(s)\|^{2} e^{2 \alpha(s-t)} d s, \quad 0 \leq t<\infty, \\
& \leq\left\|q_{0}\right\|^{2} e^{2|\alpha| T}+\frac{1}{r} e^{2|\alpha| T} \int_{0}^{T}\|f(s)\|^{2} d s, \quad 0 \leq t \leq T,
\end{aligned}
$$

where $\alpha=\frac{r}{2}+\frac{\pi \nu}{|D|}-\frac{1}{2}|\beta|\left(\frac{|D|}{\pi}+1\right)$ with $|D|$ denoting the area of the domain $D$. Note that $\alpha$ is positive in the case of no rotation $(\beta=0)$. 
Theorem 3.2. (i) Stochastic Approximation: If the stochastic parameterization $\sigma(Q, \omega)$ is such that

$$
\int_{0}^{T}\|\sigma(Q, \omega)\|^{2} d t \leq M(T), \quad \text { almost surely for } \omega \in \Omega,
$$

for some constant $M>0$ depending on the computational time interval, then

$$
\mathbb{E}\|\bar{q}-Q\|^{2} \leq C\left(\nu, r, q_{0}, T\right) \cdot \mathbb{E} \int_{0}^{T}\|R(q)-\sigma(Q, \omega)\|^{2} d t, \quad 0 \leq t \leq T,
$$

for any fixed time interval $0 \leq t \leq T$.

This implies that, if the stochastic parameterization $\sigma(Q, \omega)$ approximates the $S G S$ stress $R(q)$, then the LES solution $Q$ approximates $\bar{q}$, in the mean-square sense.

(ii) Scale convergence: If the stochastic parameterization $\sigma(Q, \omega)$ satisfies

$$
\mathbb{E} \int_{0}^{T}\|\sigma(Q, \omega)\|^{2} d t \rightarrow 0, \quad \text { as } \delta \rightarrow 0,
$$

for all LES solutions $Q$ of (2.5), then

$$
\mathbb{E}\|q-Q\|^{2} \rightarrow 0, \quad \text { as } \delta \rightarrow 0, \quad 0<t<T .
$$

This implies that, if the stochastic parameterization $\sigma(Q, \omega)$ becomes smaller (collectively in a computational time interval) as the cut-off scale size $\delta$ decreases, then the LES solution $Q$ approximates the original solution $q$ better, in the mean-square sense.

Remark 3.3. Condition (3.3) means that the stochastic parameterization $\sigma(Q, \omega)$ is square-integrable in time and space, and its norm in the space $L^{2}\left((0, T) ; L^{2}(D)\right)$ is almost surely bounded on the computational interval.

Remark 3.4. Condition (3.5) means that the variance of the stochastic parameterization $\sigma(q, \omega)$, collectively in the finite time interval of numerical simulation, becomes smaller and smaller as the cut-off scale size $\delta$ decreases.

To prove part (i), denote $U=\bar{q}-Q$, so that $U=\Delta(\bar{\psi}-\Psi)$. Note that $U(0)=0$. Subtracting (2.3) from (2.5), we see that $U$ satisfies

$$
U_{t}=-J(\bar{\psi}, \bar{q})+J(\Psi, Q)-\beta\left(\bar{\psi}_{x}-\Psi_{x}\right)+\nu \Delta U-r U+[R(q)-\sigma(Q, \omega)] .
$$

Multiplying this equation by $U$, integrating over $D$ and noting that $\bar{q}=U+Q$, we obtain

$$
\begin{aligned}
\frac{1}{2} \frac{d}{d t}\|U\|^{2}= & -\int_{D} J(\bar{\psi}-\Psi, Q) U-\beta \int_{D}\left(\bar{\psi}_{x}-\Psi_{x}\right) U \\
& -\nu\|\nabla U\|^{2}-r\|U\|^{2}+\int_{D}[R(q)-\sigma(Q, \omega)] U .
\end{aligned}
$$

Note that, for $0 \leq t \leq T$,

$$
\begin{aligned}
& \left|\int_{D} J(\bar{\psi}-\Psi, Q) U d x d y\right| \\
& \quad \leq \sqrt{\frac{2|D|}{\pi}}\|Q\|\|U\|^{2} \\
& \quad \leq \sqrt{\frac{2|D|}{\pi}}\left\{\left\|\bar{q}_{0}\right\|^{2} e^{2|\alpha| T}+\frac{1}{r} e^{2|\alpha| T} \int_{0}^{T}\left(\|\bar{f}(s)\|^{2}+\|\sigma(Q, \omega)\|^{2}\right) d s\right\}\|U\|^{2},
\end{aligned}
$$


where we used Lemma 3.1 on the LES model (2.5). Also, by the Young and Poincaré inequalities [36] we have

$$
\begin{aligned}
\left|\beta \int_{D}\left(\bar{\psi}_{x}-\Psi_{x}\right) U d x d y\right| & \leq \frac{1}{2}|\beta|\left(\int_{D}\left(\bar{\psi}_{x}-\Psi_{x}\right)^{2} d x d y+\int_{D} U^{2} d x d y\right) \\
& \leq \frac{1}{2}|\beta|\left(\frac{|D|}{\pi} \int_{D} U^{2} d x d y+\int_{D} U^{2} d x d y\right)
\end{aligned}
$$

that is,

$$
\left|\beta \int_{D}\left(\bar{\psi}_{x}-\Psi_{x}\right) U d x d y\right| \leq \frac{1}{2}|\beta|\left(\frac{|D|}{\pi}+1\right)\|U\|^{2} .
$$

Moreover,

$$
\left|\int_{D}[R(q)-\sigma(Q, \omega)] U\right| \leq \frac{1}{2}\|R(q)-\sigma(Q, \omega)\|^{2}+\frac{1}{2}\|U\|^{2} .
$$

Putting all these estimates into (3.8), we obtain

$$
\begin{aligned}
\frac{d}{d t}\|U\|^{2} \leq 2 & \left(-\alpha+\frac{1}{2}+\sqrt{\frac{2|D|}{\pi}}\right) \\
& \times\left(\left\|\bar{q}_{0}\right\|^{2} e^{2|\alpha| T}+\frac{1}{r} e^{2|\alpha| T} \int_{0}^{T}\left[\|\bar{f}(s)\|^{2}+\|\sigma(Q, \omega)\|^{2}\right] d s\right)\|U\|^{2} \\
& +\|R(q)-\sigma(Q, \omega)\|^{2} .
\end{aligned}
$$

Notice that $\alpha$ is defined in Lemma 3.1 in terms of physical parameters. By the Gronwall inequality [36] and noting $U(0)=0$, we obtain

$$
\mathbb{E}\|\bar{q}-Q\|^{2}=\mathbb{E}\|U\|^{2} \leq C\left(\nu, r, q_{0}, T\right) \cdot \mathbb{E} \int_{0}^{t}\|R(q)-\sigma(Q, \omega)\|^{2} d t, \quad 0 \leq t \leq T,
$$

where $C>0$ is a constant. This proves part (i) of Theorem 3.2.

To prove part (ii) of Theorem 3.2, denote $V=q-Q$, so that $V=\Delta(\psi-\Psi)$. Subtracting equation (2.1) from (2.5) leads to

$$
V_{t}=-J(\psi, q)+J(\Psi, Q)-\beta\left(\psi_{x}-\Psi_{x}\right)+\nu \Delta V-r V+(f-\bar{f})-\sigma(Q, \omega) .
$$

Similar to the approach in proving part (i) above, we estimate

$$
\begin{aligned}
\frac{d}{d t}\|V\|^{2} \leq 2 & \left(-\alpha+1+\sqrt{\frac{2|D|}{\pi}}\right)\left(\left\|q_{0}\right\|^{2} e^{2|\alpha| T}+\frac{1}{r} e^{2|\alpha| T} \int_{0}^{T}\|f(s)\|^{2} d s\right)\|V\|^{2} \\
& +\|f-\bar{f}\|^{2}+\|\sigma(Q, \omega)\|^{2} .
\end{aligned}
$$

By the Gronwall inequality again, we obtain

$$
\begin{aligned}
\mathbb{E}\|V\|^{2} & \leq C_{1}\left(\nu, r, q_{0}, T\right) \mathbb{E}\left\|q_{0}-\bar{q}_{0}\right\|^{2} \\
& +C_{2}\left(\nu, r, q_{0}, T\right) \cdot \mathbb{E} \int_{0}^{T}\left[\|f-\bar{f}\|^{2}+\|\sigma(Q, \omega)\|^{2}\right] d t, \quad 0 \leq t \leq T,
\end{aligned}
$$

where $C_{1}, C_{2}$ are positive constants. Due to the property of $G_{\delta}$, both $\left\|q_{0}-\bar{q}_{0}\right\|$ and $\|f-\bar{f}\|$ go to zero as $\delta \rightarrow 0$. Together with the condition (3.5), we finally see that $\mathbb{E}\|V\|^{2}=\mathbb{E}\|q-Q\|^{2} \rightarrow 0$ as $\delta \rightarrow 0$, completing the proof of Theorem 3.2. 
We view Theorem 3.2 as a starting point in our study of stochastic parameterization of SGS stress-related terms in the LES of geophysical flows in the sense that it comments on the dependence of the LES solution only on the variance of the stochastic parameterization (its closeness to that of the actual SGS stress terms). Thus, it may be argued that the stochastic nature of the parameterization is not central to the results of this paper. However, to the extent that variance is one of the most fundamental characteristics of a stochastic process, understanding the dependence of the LES solution on it is important. What is now desirable is further characterization of the dependence of the LES solution on various other aspects of the stochastic parameterization such as its temporal and spatial correlation structure and its probability distribution function. These are subjects of ongoing research and we hope to report on them in the future.

\section{ACKNOWLEDGEMENT}

This work was initiated while both authors were co-organizing the special session on Uncertainty, Random Dynamical Systems and Stochastic Modeling in Geophysics, European Geosciences Union, General Assembly, Vienna, Austria, April 2005. We thank Traian Iliescu for helpful discussions.

\section{REFERENCES}

1. L. Arnold. Hasselmann's program visited: The analysis of stochasticity in deterministic climate models. In J.-S. von Storch and P. Imkeller, editors, Stochastic climate models, pages 141-158, Boston, 2001. Birkhäuser. MR1948294 (2003j:86007)

2. Intergovernmental Panel on Climate Change (IPCC) Report on Climate Change 2001: The Scientific Basis, Section F.6., 2001.

3. P. S. Berloff. Random-forcing model of the mesoscale oceanic eddies, J. Fluid Mech. 529, 71-95, 2005.

4. P. S. Berloff, W. Dewar, S. Kravtsov, J. McWilliams and M. Ghil. Ocean eddy dynamics in a coupled ocean-atmosphere model: Diagnostics and parameterization. Preprint, 2005.

5. S. B. Pope. Turbulent Flows, Chapter 13, Cambridge University Press, 2000. MR 1881598 (2003f:76002)

6. G. D. Nastrom and K. S. Gage. A Climatology of Atmospheric Wavenumber Spectra of Wind and Temperature Observed by Commercial Aircraft, Journal of the Atmospheric Sciences: Vol. 42, No. 9, pp. 950-960, 1985.

7. C. Meneveau and J. Katz. Scale-invariance and turbulence models for Large-Eddy Simulation, Ann. Rev. Fluid Mech. 32, 1-32, 2000. MR.1744302 (2000k:76079)

8. J. Smagorinsky. General circulation experiments with the primitive equations: I. The basic experiment. Mon. Wea. Rev., 91,1963, 99-164.

9. J. Smagorinsky. Some historical remarks on the use of nonlinear viscosities. In Large Eddy Simulation of Complex Engineering and Geophysical Flows, B. Galperin and S. A. Orszag, Eds., Cambridge University Press, 1993, 3-36. MR.1248380

10. C. E. Leith. Diffusion approximation for two-dimensional turbulence. Phys. Fluids, 10, 1968, 1409-1416.

11. C. E. Leith. Stochastic models of chaotic systems. Physica D, 98, 1996, 481-491.

12. W. R. Holland. The role of mesoscale eddies in the general circulation of the ocean: Numerical experiments using a wind-driven quasi-geostrophic model. J. Phys. Oceanogr., 8, 1978, 363392.

13. R. Bleck and D. B. Boudra. Initial testing of a numerical ocean circulation model using a hybrid- (quasi-isopycnic) vertical coordinate. J. Phys. Oceanogr., 11, 1981, 755-770.

14. A. J. Majda, I. Timofeyev and E. Vanden-Eijnden. Models for stochastic climate prediction, Proc. National Acad. Sci. 96, 1999, 14687-14691. MR1731439 (2000h:86007)

15. A. J. Majda, I. Timofeyev and E. Vanden-Eijnden. Systematic Strategies for Stochastic Mode Reduction in Climate, Journal of the Atmospheric Sciences: Vol. 60, No. 14, pp. 1705-1722. MR 2030132 (2004j:86011) 
16. J. R. Herring. Stochastic Modeling of Turbulent Flows. In Stochastic Modeling in Physical Oceanography, A. Adler, B.M. Culler, Eds., Birkhäuser, 1996. MR1383874 (96k:76061)

17. R. H. Kraichnan. The structure of isotropic turbulence at very high Reynolds number. $J$. Fluid Mech. 5, 497-543, 1959. MR0105963 (21:4697)

18. A. S. Monin and A. M. Yaglom. Statistical Fluid Mechanics, Vol. 1, The MIT Press, 769 pp., 1975.

19. L. C. Berselli, G. P. Galdi, T. Iliescu and W. Layton. Mathematical analysis for the rational large eddy simulation model. Math. Models Methods Appl. Sci. 12 (2002), no. 8, 1131-1152. MR.1924604 (2003k:76077)

20. J. Duan and P. E. Kloeden. Dissipative Quasigeostrophic Motion under Temporally Almost Periodic Forcing, J. Math. Anal. Appl. 236 (1999), 74-85. MR1702691(2001j:76115)

21. D. Blomker, J. Duan and T. Wanner. Enstrophy Dynamics of Stochastically Forced LargeScale Geophysical Flows. J. Math. Phys., 43 (2002), 2616-2626. MR.1893690 (2003a:76017)

22. P. Constantin and C. Foias. Navier-Stokes Equations, Univ. of Chicago Press, Chicago, 1988. MR0972259 (90b:35190)

23. J. Duan, P. E. Kloeden and B. Schmalfuss. Exponential Stability of the Quasigeostrophic Equation under Random Perturbations. Progress in Probability 49(2000), 241256. MR1948299 (2004b:76066)

24. J. Duan and B. Goldys. Ergodicity of Stochastically Forced Large Scale Geophysical Flows. Inter. J. Math. Math. Sci., 28 (2001), 313-320. MR1887567(2002k:37163)

25. V. P. Dymnikov and A. N. Filatov. Mathematics of Climate Modeling, Birkhäuser, Boston, 1997. MR $1426827(99 \mathrm{k}: 76058)$

26. T. DelSole and B. F. Farrell. A stochastically excited linear system as a model for quasigeostrophic turbulence: Analytic results for one- and two-layer fluids, J. Atmos. Sci. 52 (1995) 2531-2547.

27. A. E. Gill. Atmosphere-Ocean Dynamics. Academic Press, New York, 1982.

28. K. Hasselmann. Stochastic climate models: Part I. Theory, Tellus 28 (1976), 473-485.

29. M. Kaya and W. Layton. On verification of models of the motion of large eddies in turbulent flows. Differential and Integral Equations 15 (2002), 1395-1407. MR.1920694 (2003f:76059)

30. B. T. Nadiga, D. Livescu and C.Q. McKay. Stochastic Large Eddy Simulation of Geostrophic Turbulence, Eos. Trans. AGU, 86(18), Jt. Assem. Suppl., Abstract NG23A-09, 2005.

31. B. T. Nadiga, D. Livescu, and C.Q. McKay. Geophysical Research Abstracts, Vol. 7, 05488, 2005.

32. R. J. Greatbatch and B. T. Nadiga. Four gyre circulation in a barotropic model with double gyre wind forcing, J. Phys. Ocean., 30 (2000), 1461-1471.

33. B. Oksendal. Stochastic Differential Equations. Sixth Ed., Springer-Verlag, New York, 2003. MR2001996 (2004e:60102)

34. J. Pedlosky. Geophysical Fluid Dynamics, Springer-Verlag, 2nd edition, 1987.

35. B. L. Rozovskii. Stochastic Evolution Equations. Kluwer Academic Publishers, Boston, 1990. MR.1135324 (92k:60136)

36. R. Temam. Infinite-Dimensional Dynamical Systems in Mechanics and Physics, SpringerVerlag, New York, 1988. MR0953967 (89m:58056)

Department of Applied Mathematics, Illinois Institute of Technology, Chicago, IlliNOIS 60616

E-mail address: duan@iit.edu

Los Alamos National Laboratory, MS-B296, Los Alamos, New Mexico 87545

E-mail address: balu@lanl.gov 